\title{
End Point Results in Metric Spaces Endowed with a Graph
}

\author{
Binayak S. Choudhury, ${ }^{1}$ Nikhilesh Metiya, ${ }^{2}$ and Pradip Debnath ${ }^{3}$ \\ ${ }^{1}$ Department of Mathematics, Indian Institute of Engineering Science and Technology, Shibpur, Howrah, West Bengal 711103, India \\ ${ }^{2}$ Department of Mathematics, Sovarani Memorial College, Jagatballavpur, Howrah, West Bengal 711408, India \\ ${ }^{3}$ Department of Mathematics, North Eastern Regional Institute of Science and Technology, Nirjuli, Arunachal Pradesh 791109, India
}

Correspondence should be addressed to Nikhilesh Metiya; metiya.nikhilesh@gmail.com

Received 23 July 2016; Accepted 3 October 2016

Academic Editor: Krassimir T. Atanassov

Copyright (C) 2016 Binayak S. Choudhury et al. This is an open access article distributed under the Creative Commons Attribution License, which permits unrestricted use, distribution, and reproduction in any medium, provided the original work is properly cited.

We introduce the notion of end point of multivalued mappings in the setting of metric space endowed with a graph and prove some existence results in this context. The mappings are assumed to satisfy certain generalized multivalued almost $G$-contractive type inequalities. Further, the consequences of the corresponding results in the cases of single-valued mappings are also discussed with examples.

\section{Introduction and Mathematical Preliminaries}

One of the most famous fixed point theorems is the Banach contraction principle, which initiated a new era of research in fixed point theory due to its immense applicability in major areas of mathematics like numerical analysis and differential/integral equations. This important principle was used by Boyd and Wong [1] to investigate the fixed point results of nonlinear contraction maps.

Study of fixed point results in partially ordered sets has been a very well-motivated area of research because of its ease of compatibility in modelling various problems and in finding new convergence schemes. The first attempt in this direction was carried out by Ran and Reurings [2] where they combined the Banach Contraction Principle and the KnasterTarski fixed point theorem. Ran and Reurings considered a class of mappings $f: X \rightarrow X$, with $(X, d)$ as a complete metric space and a partial order $\leq$. The mappings they considered were continuous, monotone with respect to the partial order $\leq$. Those mappings also satisfy a Banach contraction inequality for every pair $(x, y) \in X \times X$ such that $x \leq y$. When for some $x_{0} \in X$, the inequality $x_{0} \leq f\left(x_{0}\right)$ is satisfied, they proved that the Picard sequence $\left\{f^{n}\left(x_{0}\right)\right\}$ would converge to a fixed point of $f$. Ran and Reurings also combined this interesting result with the Schauder fixed point theorem and applied it to obtain some existence and uniqueness results to nonlinear matrix equations.

Neito and Rodríguez-López $([3,4])$ extended the results of Ran and Reurings to the functions which were not necessarily continuous. They also applied their results to obtain a theorem on the existence of a unique solution for periodic boundary problems relative to ordinary differential equations.

The additional structure of ordering was introduced in metrizable uniform spaces by Turinici [5]. Later, fixed point studies were attempted in partially ordered metric spaces in a good number of works as, for instance, in [6-9]. Multivalued fixed point results were deduced in such contexts in works like [10-14].

The use of graph was introduced in metric fixed point theory by Jachymski [15]. It is in furtherance of metric fixed point theory in partially ordered spaces in that the partial orders themselves introduce a directed graph in metric spaces. A version of the Banach contraction mapping principle was established in these spaces where contraction inequality needs to be satisfied on the edges of the graph. In fact this is a general feature of the line of research which is that the contractions can be restricted to parts of spaces for the purpose of fixed points to exist. There are several results on fixed points 
of functions defined on these structures, some of the important references from these works being [16-20].

In the following we describe the mathematical background materials which are necessary for establishing the results in this papers.

Let $(X, d)$ be a metric space. We consider the following classes of subsets of the metric space $X$ :

$$
\begin{aligned}
& N(X)=\{A: A \text { is a non-empty subset of } X\}, \\
& B(X) \\
& \quad=\{A: A \text { is a non-empty bounded subset of } X\} .
\end{aligned}
$$

For $A, B \in B(X)$, the functions $D$ and $\delta$ are defined as follows:

$$
\begin{aligned}
& D(A, B)=\inf \{d(a, b): a \in A, b \in B\}, \\
& \delta(A, B)=\sup \{d(a, b): a \in A, b \in B\} .
\end{aligned}
$$

If $A=\{a\}$, then we write $D(A, B)=D(a, B)$ and $\delta(A, B)=$ $\delta(a, B)$. Also in addition, if $B=\{b\}$, then $D(A, B)=d(a, b)$ and $\delta(A, B)=d(a, b)$. Obviously, $D(A, B) \leq \delta(A, B)$. For all $A, B, C \in B(X)$, the definition of $\delta(A, B)$ yields the following:

$$
\begin{aligned}
& \delta(A, B)=\delta(B, A), \\
& \delta(A, B) \leq \delta(A, C)+\delta(C, B), \\
& \delta(A, B)=0, \quad \text { iff } A=B=\{a\}, \\
& \delta(A, A)=\operatorname{diam} A
\end{aligned}
$$

(see [21]) $\delta$-distance is not a metric like the Hausdorff distance [22] but shares all the properties of a metric except that it is possible to have $\delta(A, A) \neq 0$.

Definition 1. Let $X$ be a nonempty set and $T$ a self-map on $X$. By a fixed point of $T$, we mean an element $x$ of $X$ such that $x=T x$. The set of all fixed points of $T$ is denoted as $\operatorname{Fix}(T)$.

Definition 2. Let $X$ be a nonempty set and $T: X \rightarrow N(X)$ be multivalued mapping. Then $x \in X$ is called a fixed point of $T$ if $x \in T x$.

Definition 3. Let $X$ be a nonempty set and $T: X \rightarrow N(X)$ be multivalued mapping. Then $x \in X$ is called an end point of $T$ if $\{x\}=T x$. The set of all end points of $T$ is denoted as $\operatorname{End}(T)$.

Remark 4. Every end point of a mapping $T: X \rightarrow N(X)$ is a fixed point of the mapping $T$ but the converse is not true. So $\operatorname{End}(T) \subseteq \operatorname{Fix}(T)$.

Fixed point theory for multivalued operators is an important topic of set-valued analysis. There are several works on fixed point theory of set-valued maps which have utilized $\delta$ distance [10-13, 21, 23, 24].

Berinde [25] introduced a new class of self-mappings (usually almost contractions) that satisfy a simple but general contraction condition that includes any strict contraction, Kannan [26] and Zamfirescu [27] mappings, and a large class of quasi-contractions. Almost contractions and their generalizations were further considered in several works like $[12,13,28-31]$. Our aim in this paper is to deduce some fixed point theorems for certain multivalued almost contractions using $\delta$-distance.

Let $X$ be a nonempty set and $\Delta:=\{(x, x): x \in X\}$. Let $G$ be a directed graph such that its vertex set $V(G)$ coincides with $X$; that is, $V(G)=X$ and the edge set $E(G)$ contains all loops; that is, $\Delta \subseteq E(G)$. Assume that $G$ has no parallel edges. We can identify $G$ with the pair $G(V(G), E(G))$. By $G^{-1}$ we denote the conversion of a graph $G$, that is, the graph obtained from $G$ by reversing the directions of the edges. Thus we have

$$
\begin{aligned}
& V\left(G^{-1}\right)=V(G), \\
& E\left(G^{-1}\right)=\{(x, y) \in X \times X:(y, x) \in E(G)\} .
\end{aligned}
$$

Let $\widetilde{G}$ denote the undirected graph obtained from $G$ by ignoring the direction of edges. Actually, it will be more convenient for us to treat $\widetilde{G}$ as a directed graph for which the set of its edges is symmetric. Under this convention,

$$
\begin{aligned}
& V(\widetilde{G})=V(G), \\
& E(\widetilde{G})=E(G) \cup E\left(G^{-1}\right) .
\end{aligned}
$$

A graph $S(V(S), E(S))$ is called a subgraph of the graph $G(V(G), E(G))$ if $V(S) \subseteq V(G)$ and $E(S) \subseteq E(G)$.

Definition 5. If $x$ and $y$ are vertices in a graph $G$, then a path in $G$ from $x$ to $y$ of length $m(m \in \mathbb{N})$ is a sequence $\left(x_{i}\right)_{i=0}^{m}$ of $m+1$ vertices such that $x_{0}=x, x_{m}=y$, and $\left(x_{i-1}, x_{i}\right) \in E(G)$ for $i=1, \ldots, m$.

A graph $G$ is connected if there is a path between any two vertices. $G$ is weakly connected if $\widetilde{G}$ is connected. If $G$ is such that $E(G)$ is symmetric and $x$ is a vertex in $G$, then the subgraph $G_{x}$ consisting of all edges and vertices which are contained in some path beginning at $x$ is called the component of $G$ containing $x$. In this case $V\left(G_{x}\right)=[x]_{G}$, where $[x]_{G}$ is the equivalence class of the following relation $R$ defined on $V(G)$ by the rule:

$$
y R z \text { if there is a path in } G \text { from } y \text { to } z \text {. }
$$

Definition 6. Let $T: X \rightarrow X$ be a mapping. Then $\operatorname{Fix}(T)$, $X_{T}(G), X^{T}(G)$, and $X_{T}^{T}(G)$ are defined as follows:

$$
\begin{aligned}
\operatorname{Fix}(T) & :=\{x \in X: x=T x\} \\
X_{T}(G) & :=\{x \in X:(x, T x) \in E(G)\}, \\
X^{T}(G) & :=\{x \in X:(T x, x) \in E(G)\}, \\
X_{T}^{T}(G) & :=\{x \in X:(x, T x),(T x, x) \in E(G)\} \\
& =X_{T}(G) \cap X^{T}(G) .
\end{aligned}
$$


Definition 7. Let $T: X \rightarrow N(X)$ be a multivalued mapping. Then $\operatorname{Fix}(T), \operatorname{End}(T), X_{T}(G), X^{T}(G)$, and $X_{T}^{T}(G)$ are defined as follows:

$$
\begin{aligned}
& \text { Fix }(T):=\{x \in X: x \in T x\}, \\
& \text { End }(T):=\{x \in X:\{x\}=T x\}, \\
& X_{T}(G):=\{x \in X:(x, u) \in E(G) \text { for some } u \in T x\}, \\
& X^{T}(G):=\{x \in X:(u, x) \in E(G) \text { for some } u \in T x\}, \\
& X_{T}^{T}(G) \\
& \quad:=\{x \in X:(x, u),(u, x) \in E(G) \text { for some } u \in T x\} \\
& \quad=X_{T}(G) \cap X^{T}(G) .
\end{aligned}
$$

The essential feature of Jachymski's work [15] is that the contraction inequality needs to be satisfied only on certain edges of the graph. This opened a new direction in fixed point theory in which a significant amount of works has appeared. Our work is in furtherance of this line of research.

\section{Main Results}

We say a metric space $(X, d)$ is endowed with a directed graph $G$, if $G$ is a directed graph such that $V(G)=X$ and $\Delta \subseteq E(G)$.

In the following we define generalized almost $G$ contraction for single-valued mappings and generalized multivalued almost $G$-contraction of types (A), (B), and (C). Assume that $(X, d)$ is a metric space, and $G$ is a directed graph such that $V(G)=X$ and $\Delta \subseteq E(G)$.

Definition 8. A mapping $T: X \rightarrow X$ is a generalized almost $G$-contraction if for all $x, y \in X$ with $(x, y) \in E(G)$

(1) $(T x, T y) \in E(G)$,

(2) $d(T x, T y) \leq \alpha \max \{d(x, y), d(x, T x), d(y, T y),(d(x$, $T y)+d(y, T x)) / 2\}+L \min \{d(x, T x), d(y, T y), d(x$, $T y), d(y, T x)\}$

where $\alpha \in(0,1)$ and $L \geq 0$.

Definition 9. A mapping $T: X \rightarrow B(X)$ is a generalized multivalued almost $G$-contraction of type (A) if for all $x, y \in$ $X$ with $(x, y) \in E(G)$

(i) $u \in T x$ implies that there exists $v \in T y$ such that $(u, v) \in E(G)$

(ii) $\delta(T x, T y) \leq \alpha \max \{d(x, y), D(x, T x), D(y, T y)$, $(D(x, T y)+D(y, T x)) / 2\}+L \min \{D(x, T x), D(y, T y)$, $D(x, T y), D(y, T x)\}$,

where $\alpha \in(0,1)$ and $L \geq 0$.

Definition 10. A mapping $T: X \rightarrow B(X)$ is a generalized multivalued almost $G$-contraction of type (B) if for all $x, y \in$ $X$ with $(x, y) \in E(G)$

(i) $v \in T y$ implies that there exists $u \in T x$ such that $(u, v) \in E(G)$ (ii) $\delta(T x, T y) \leq \alpha \max \{d(x, y), D(x, T x), D(y, T y)$, $(D(x, T y)+D(y, T x)) / 2\}+L \min \{D(x, T x), D(y, T y)$, $D(x, T y), D(y, T x)\}$,

where $\alpha \in(0,1)$ and $L \geq 0$.

Definition 11. A mapping $T: X \rightarrow B(X)$ is a generalized multivalued almost $G$-contraction of type (C) if for all $x, y \in$ $X$ with $(x, y) \in E(G)$

(i) $u \in T x$ implies that there exists $v \in T y$ such that $(u, v) \in E(G)$ and $v \in T y$ implies that there exists $u \in T x$ such that $(u, v) \in E(G)$,

(ii) $\delta(T x, T y) \leq \alpha \max \{d(x, y), D(x, T x), D(y, T y)$, $(D(x, T y)+D(y, T x)) / 2\}+L \min \{D(x, T x), D(y, T y)$, $D(x, T y), D(y, T x)\}$,

where $\alpha \in(0,1)$ and $L \geq 0$.

Proposition 12. If $T$ is generalized multivalued almost Gcontraction of type $(B)$ then $T$ is generalized multivalued almost $G^{-1}$-contraction of type $(A)$.

Proof. Suppose that $T$ is generalized multivalued almost $G$ contraction of type (B). Let $x, y \in X$ with $(x, y) \in E(G)$. Then $(y, x) \in E\left(G^{-1}\right)$.

(1) By the condition (i) of the Definition 10,v $\in$ Ty implies that there exists $u \in T x$ such that $(u, v) \in$ $E(G)$. Now $(u, v) \in E(G)$ implies that $(v, u) \in E\left(G^{-1}\right)$. Therefore, if $x, y \in X$ with $(y, x) \in E\left(G^{-1}\right)$, then $v \in T y$ implies that there exists $u \in T x$ such that $(v, u) \in E\left(G^{-1}\right)$.

(2) By the condition (ii) of the Definition 10, for all $x, y \in$ $X$ with $(x, y) \in E(G)$

$$
\begin{aligned}
& \delta(T x, T y) \leq \alpha \max \{d(x, y), D(x, T x), D(y, T y), \\
& \left.\frac{D(x, T y)+D(y, T x)}{2}\right\}+L \min \{D(x, T x), \\
& D(y, T y), D(x, T y), D(y, T x)\},
\end{aligned}
$$

where $\alpha \in(0,1)$ and $L \geq 0$.

Since $(x, y) \in E(G)$ implies that $(y, x) \in E\left(G^{-1}\right)$ and $\delta$ and $d$ are symmetric, it follows that for all $x, y \in X$ with $(y, x) \in$ $E\left(G^{-1}\right)$

$$
\begin{aligned}
& \delta(T y, T x) \leq \alpha \max \{d(y, x), D(y, T y), D(x, T x), \\
& \left.\frac{D(y, T x)+D(x, T y)}{2}\right\}+L \min \{D(y, T y), \\
& D(x, T x), D(y, T x), D(x, T y)\},
\end{aligned}
$$

where $\alpha \in(0,1)$ and $L \geq 0$.

Then it follows by Definition 9 that $T$ is generalized multivalued almost $G^{-1}$-contraction of type (A). 
Proposition 13. If T is generalized multivalued almost G-contraction of type $(C)$ then $T$ is generalized multivalued almost $\widetilde{G}$-contraction of type $(A)$.

Proof. Suppose that $T$ is generalized multivalued almost Gcontraction of type (C). By Definitions 9 and 10 and Proposition 12 it follows that $T$ is both generalized multivalued almost $G$-contraction of type (A) and generalized multivalued almost $G^{-1}$-contraction of type (A). Hence $T$ is generalized multivalued almost $\widetilde{G}$-contraction of type (A).

Definition 14. The triple $(X, d, G)$ is said to be regular if

(1) for any sequence $\left\{x_{n}\right\}$ in $X$ with $x_{n} \rightarrow x$ and $\left(x_{n}, x_{n+1}\right) \in E(G)$ for all $n \in \mathbb{N},\left(x_{n}, x\right) \in E(G)$ for all $n \in \mathbb{N}$,

(2) for any sequence $\left\{x_{n}\right\}$ in $X$ with $x_{n} \rightarrow x$ and $\left(x_{n+1}, x_{n}\right) \in E(G)$ for all $n \in \mathbb{N},\left(x, x_{n}\right) \in E(G)$ for all $n \in \mathbb{N}$.

Theorem 15. Let $(X, d)$ be a complete metric space endowed with a directed graph $G$ and $T: X \rightarrow B(X)$ be a generalized multivalued almost $G$-contraction of type $(A)$. Suppose that the triple $(X, d, G)$ is regular. Then the following statements hold:

(1) For any $x \in X_{T}(G),\left.T\right|_{[x]_{\widetilde{G}}}$ has an end point.

(2) If $X_{T}(G) \neq \emptyset$ and $G$ is weakly connected, then $T$ has an end point in $X$.

(3) If $X^{*}:=\bigcup\left\{[x]_{\widetilde{G}}: x \in X_{T}(G)\right\}$, then $\left.T\right|_{X^{*}}$ has an end point.

(4) $\operatorname{End}(T) \neq \emptyset$ if and only if $X_{T}(G) \neq \emptyset$.

Proof. (1) Let $x_{0} \in X_{T}(G)$. By the definition of $X_{T}(G)$, there exists $x_{1} \in T x_{0}$ such that $\left(x_{0}, x_{1}\right) \in E(G)$. Since $T$ is generalized multivalued almost $G$-contraction of type (A), there exists $x_{2} \in T x_{1}$ such that $\left(x_{1}, x_{2}\right) \in E(G)$. Again, by similar logic, there exists $x_{3} \in T x_{2}$ such that $\left(x_{2}, x_{3}\right) \in E(G)$. Continuing this process we construct a sequence $\left\{x_{n}\right\}$ in $X$ such that $\forall n \geq 0$

$$
\begin{gathered}
x_{n+1} \in T x_{n}, \\
\left(x_{n}, x_{n+1}\right) \in E(G) .
\end{gathered}
$$

Since $\left(x_{n}, x_{n+1}\right) \in E(G)$ and $T$ is generalized multivalued almost $G$-contraction of type (A), we have, for all $n \geq 0$,

$$
\begin{aligned}
& d\left(x_{n+1}, x_{n+2}\right) \leq \delta\left(T x_{n}, T x_{n+1}\right) \leq \alpha \max \left\{d\left(x_{n}, x_{n+1}\right),\right. \\
& D\left(x_{n}, T x_{n}\right), D\left(x_{n+1}, T x_{n+1}\right), \\
& \left.\frac{D\left(x_{n}, T x_{n+1}\right)+D\left(x_{n+1}, T x_{n}\right)}{2}\right\}+L \\
& \cdot \min \left\{D\left(x_{n}, T x_{n}\right), D\left(x_{n+1}, T x_{n+1}\right), D\left(x_{n}, T x_{n+1}\right),\right.
\end{aligned}
$$

$$
\begin{aligned}
& \left.D\left(x_{n+1}, T x_{n}\right)\right\} \leq \alpha \max \left\{d\left(x_{n}, x_{n+1}\right), d\left(x_{n}, x_{n+1}\right),\right. \\
& \left.d\left(x_{n+1}, x_{n+2}\right), \frac{d\left(x_{n}, x_{n+2}\right)+d\left(x_{n+1}, x_{n+1}\right)}{2}\right\}+L \\
& \cdot \min \left\{d\left(x_{n}, x_{n+1}\right), d\left(x_{n+1}, x_{n+2}\right), d\left(x_{n}, x_{n+2}\right),\right. \\
& \left.d\left(x_{n+1}, x_{n+1}\right)\right\} \leq \alpha \max \left\{d\left(x_{n}, x_{n+1}\right),\right. \\
& \left.d\left(x_{n+1}, x_{n+2}\right), \frac{d\left(x_{n}, x_{n+2}\right)}{2}\right\} .
\end{aligned}
$$

Since $d\left(x_{n}, x_{n+2}\right) / 2 \leq \max \left\{d\left(x_{n}, x_{n+1}\right), d\left(x_{n+1}, x_{n+2}\right)\right\}$, it follows that

$$
\begin{aligned}
d\left(x_{n+1}, x_{n+2}\right) & \leq \delta\left(T x_{n}, T x_{n+1}\right) \\
& \leq \alpha \max \left\{d\left(x_{n}, x_{n+1}\right), d\left(x_{n+1}, x_{n+2}\right)\right\} .
\end{aligned}
$$

Suppose that $d\left(x_{n}, x_{n+1}\right)<d\left(x_{n+1}, x_{n+2}\right)$, for some positive integer $n$. So, $0<d\left(x_{n+1}, x_{n+2}\right)$.

Then it follows from (13) that

$$
0<d\left(x_{n+1}, x_{n+2}\right) \leq \alpha d\left(x_{n+1}, x_{n+2}\right),
$$

which is a contradiction since $\alpha \in(0,1)$. Therefore, $d\left(x_{n+1}\right.$, $\left.x_{n+2}\right) \leq d\left(x_{n}, x_{n+1}\right)$, for all $n \geq 0$ and hence we have from (13) that

$$
d\left(x_{n+1}, x_{n+2}\right) \leq \delta\left(T x_{n}, T x_{n+1}\right) \leq \alpha d\left(x_{n}, x_{n+1}\right) .
$$

By repeated application of (15), we have

$$
d\left(x_{n+1}, x_{n+2}\right) \leq \alpha^{n+1} d\left(x_{0}, x_{1}\right) .
$$

For arbitrary $m, n \in \mathbb{N}$ with $m>n$,

$$
\begin{aligned}
& d\left(x_{m}, x_{n}\right) \\
& \leq d\left(x_{n}, x_{n+1}\right)+d\left(x_{n+1}, x_{n+2}\right)+\cdots \\
& \quad+d\left(x_{m-1}, x_{m}\right) \\
& \leq\left[\alpha^{n}+\alpha^{n+1}+\alpha^{n+2}+\cdots+\alpha^{m-1}\right] d\left(x_{0}, x_{1}\right) \\
& \leq \frac{\alpha^{n}}{1-\alpha} d\left(x_{0}, x_{1}\right) \longrightarrow 0, \\
& \text { as } n \longrightarrow \infty \text { (since } \alpha \in(0,1)),
\end{aligned}
$$

which implies that $\left\{x_{n}\right\}$ is a Cauchy sequence. From the completeness of $X$, there exists a $z \in X$ such that

$$
x_{n} \longrightarrow z, \quad \text { as } n \longrightarrow \infty \text {. }
$$

Since $\left\{x_{n}\right\}$ is a sequence in $X$ such that $x_{n} \rightarrow z$ and $\left(x_{n}, x_{n+1}\right) \in E(G)$ for all $n \in \mathbb{N}$, using the regular property of $(X, d, G)$ we have that

$$
\left(x_{n}, z\right) \in E(G), \quad \forall n \geq 0 .
$$


Since $\left(x_{n}, z\right) \in E(G)$ for all $n \geq 0$ and $T$ is generalized multivalued almost $G$-contraction of type (A), we have

$$
\begin{aligned}
& \delta\left(x_{n+1}, T z\right) \leq \delta\left(T x_{n}, T z\right) \leq \alpha \max \left\{d\left(x_{n}, z\right),\right. \\
& \left.D\left(x_{n}, T x_{n}\right), D(z, T z), \frac{D\left(x_{n}, T z\right)+D\left(z, T x_{n}\right)}{2}\right\} \\
& +L \min \left\{D\left(x_{n}, T x_{n}\right), D(z, T z), D\left(x_{n}, T z\right),\right. \\
& \left.D\left(z, T x_{n}\right)\right\} \leq \alpha \max \left\{d\left(x_{n}, z\right), d\left(x_{n}, x_{n+1}\right),\right. \\
& \left.D(z, T z), \frac{D\left(x_{n}, T z\right)+d\left(z, x_{n+1}\right)}{2}\right\}+L \\
& \cdot \min \left\{d\left(x_{n}, x_{n+1}\right), D(z, T z), D\left(x_{n}, T z\right)\right. \\
& \left.d\left(z, x_{n+1}\right)\right\} .
\end{aligned}
$$

Taking the limit as $n \rightarrow \infty$ in the above inequality and using (18), we have

$$
\delta(z, T z) \leq \alpha D(z, T z) \leq \alpha \delta(z, T z) .
$$

Since $\alpha \in(0,1)$, the above inequality implies that $\delta(z, T z)=0$ or that $\{z\}=T z$. Moreover, $z$ is an end point of $T$. By (19), it is obvious that $\left(x_{0}, z\right) \in E(G)$ and so $z \in\left[x_{0}\right]_{\widetilde{G}}$. Hence $\left.T\right|_{\left[x_{0}\right]_{\widetilde{G}}}$ has an end point.

(2) Let $X_{T}(G) \neq \emptyset$ and $G$ is weakly connected. Since $G$ is weakly connected, $[x]_{\widetilde{G}}=X$ for every $x \in X$. Since $X_{T}(G) \neq$ $\emptyset$, there exists $x_{0} \in X_{T}(G)$. Then $\left[x_{0}\right]_{\widetilde{G}}=X$. So by (1), $T$ has an end point in $X$.

(3) Let $X^{*}:=\bigcup\left\{[x]_{\widetilde{G}}: x \in X_{T}(G)\right\}$. By (1) and (2), T| $\left.\right|_{X^{*}}$ has an end point.

(4) Let $\operatorname{End}(T) \neq \emptyset$. Then there exists at least one element $x \in \operatorname{End}(T)$. Now $x \in \operatorname{End}(T)$ means $\{x\}=T x$. So, $x \in T x$. Now $\Delta \subseteq E(G)$ implies that $(x, x) \in E(G)$. Therefore, we have $x \in T x$ and $(x, x) \in E(G)$, which implies that $x \in X_{T}(G)$. Hence $X_{T}(G) \neq \emptyset$. Conversely suppose that $X_{T}(G) \neq \emptyset$. Then by $(1), \operatorname{End}(T) \neq \emptyset$.

Theorem 16. Let $(X, d)$ be a complete metric space and $T$ : $X \rightarrow B(X)$ be a generalized multivalued almost $G$-contraction of type (B). Suppose that the triple $(X, d, G)$ is regular. Then the following statements hold:

(1) For any $x \in X^{T}(G),\left.T\right|_{[x]_{\widetilde{G}}}$ has an end point.

(2) If $X^{T}(G) \neq \emptyset$ and $G$ is weakly connected, then $T$ has an end point in $X$.

(3) If $X^{*}:=\bigcup\left\{[x]_{\widetilde{G}}: x \in X^{T}(G)\right\}$, then $\left.T\right|_{X^{*}}$ has an end point.

(4) $\operatorname{End}(T) \neq \emptyset$ if and only if $X^{T}(G) \neq \emptyset$.

Proof. Let $x \in X^{T}(G)$. Then there exist $u \in T x$ such that $(u, x) \in E(G)$. Now $(u, x) \in E(G)$ implies that $(x, u) \in$ $E\left(G^{-1}\right)$. Since $x \in X$ and $(x, u) \in E\left(G^{-1}\right)$ for some $u \in T x$, we have $x \in X_{T}\left(G^{-1}\right)$. Conversely, $y \in X_{T}\left(G^{-1}\right)$ implies that $y \in X^{T}(G)$. So $X^{T}(G)=X_{T}\left(G^{-1}\right)$. Therefore,

(1) $X^{T}(G) \neq \emptyset$ implies that $X_{T}\left(G^{-1}\right) \neq \emptyset$,

(2) $G$ is weakly connected implies that $G^{-1}$ is also weakly connected,

(3) $[x]_{\widetilde{G}}=[x]_{\widetilde{G^{-1}}}$,

(4) $X^{*}:=\bigcup\left\{[x]_{\widetilde{G}}: x \in X^{T}(G)\right\}=\bigcup\left\{[x]_{\widetilde{G^{-1}}}: x \in\right.$ $\left.X_{T}\left(G^{-1}\right)\right\}$,

(5) By Proposition 12, $T: X \rightarrow B(X)$ is a generalized multivalued almost $G^{-1}$-contraction of type (A). proof.

Let $G^{-1}=W$. Then by Theorem 15 we have the required

Theorem 17. Let $(X, d)$ be a complete metric space and $T$ : $X \rightarrow B(X)$ be a generalized multivalued almost $G$-contraction of type $(C)$. Suppose that the triple $(X, d, G)$ is regular. Then the following statements hold:

(1) For any $x \in X_{T}^{T}(G),\left.T\right|_{[x]_{\widetilde{G}}}$ has an end point.

(2) If $X_{T}^{T}(G) \neq \emptyset$ and $G$ is weakly connected, then $T$ has an end point in $X$.

(3) If $X^{*}:=\bigcup\left\{[x]_{\widetilde{G}}: x \in X_{T}^{T}(G)\right\}$, then $\left.T\right|_{X^{*}}$ has an end point.

(4) $\operatorname{End}(T) \neq \emptyset$ if and only if $X_{T}^{T}(G) \neq \emptyset$.

Proof. Arguing similarly as in the proof of Theorem 16, we have that $X_{T}^{T}(G)=X_{T}(\widetilde{G})$. Therefore,

(1) $X_{T}^{T}(G) \neq \emptyset$ implies that $X_{T}(\widetilde{G}) \neq \emptyset$,

(2) $G$ is weakly connected implies that $\widetilde{G}$ is connected,

(3) $X^{*}:=\bigcup\left\{[x]_{\widetilde{G}}: x \in X_{T}^{T}(G)\right\}=\bigcup\left\{[x]_{\widetilde{G}}: x \in X_{T}(\widetilde{G})\right\}$,

(4) By Proposition 13, $T: X \rightarrow B(X)$ is a generalized multivalued almost $\widetilde{G}$-contraction of type $(A)$.

Let $\widetilde{G}=W$. Then by Theorem 15 we have the required proof.

Example 18. Let $X=\left\{v_{1}=(0,0), v_{2}=(-1 / 2,-1 / 2), v_{3}=\right.$ $(0,-1)\}$ be a subset of $R^{2}$ ( $R$ is the set of all real numbers) and $G$ be a directed graph with $V(G)=X$ and $E(G)=$ $\left\{\left(v_{1}, v_{1}\right),\left(v_{2}, v_{2}\right),\left(v_{3}, v_{3}\right),\left(v_{2}, v_{1}\right),\left(v_{3}, v_{1}\right)\right\}$. Let $d$ be metric on $X$ defined as

$$
\begin{aligned}
& d(x, y)=\max \{|p-u|,|q-v|\}, \\
& \qquad \text { for } x=(p, q), y=(u, v) \in X .
\end{aligned}
$$

Let $T: X \rightarrow B(X)$ be defined as follows:

$$
T x= \begin{cases}\left\{v_{1}\right\}, & \text { if } x=v_{1}, \\ \left\{v_{1}, v_{2}\right\}, & \text { if } x=v_{3}, \\ \left\{v_{1}\right\}, & \text { if } x=v_{2} .\end{cases}
$$

Let $L>1$ and $\alpha \in(1 / 2,1)$.

All the conditions of Theorem 15 are satisfied and it is seen that $v_{1}$ is an end point of $T$. 


\section{Consequences in Single-Valued Cases}

In this section we obtain some consequences of the corresponding results of Section 2 in the cases of single-valued mappings.

Theorem 19. Let $(X, d)$ be a complete metric space and $T$ : $X \rightarrow X$ be a generalized almost G-contraction. Suppose that the triple $(X, d, G)$ is regular. Then following statements hold:

(1) For any $x \in X_{T}(G),\left.T\right|_{[x]_{\widetilde{G}}}$ has a fixed point.

(2) If $X_{T}(G) \neq \emptyset$ and $G$ is weakly connected, then $T$ has a fixed point in $X$.

(3) If $X^{*}:=\bigcup\left\{[x]_{\widetilde{G}}: x \in X_{T}(G)\right\}$, then $\left.T\right|_{X^{*}}$ has a fixed point.

(4) $\operatorname{Fix}(T) \neq \emptyset$ if and only if $X_{T}(G) \neq \emptyset$.

Proof. We know that, for every $x \in X,\{x\} \in B(X)$. We define a multivalued mapping $S: X \rightarrow B(X)$ as $S x=\{T x\}$, for $x \in X$.

(1) Since $T: X \rightarrow X$ is a generalized almost $G$ contraction, it follows by Definitions 8 and 9 that $S$ is a generalized multivalued almost $G$-contraction of type $(A)$.

(2) $X_{T}(G)=X_{S}(G)$. So, $X_{T}(G) \neq \emptyset$ implies that $X_{S}(G) \neq$ $\emptyset$.

(3) $X^{*}:=\bigcup\left\{[x]_{\widetilde{G}}: x \in X_{T}(G)\right\}=\bigcup\left\{[x]_{\widetilde{G}}: x \in X_{S}(G)\right\}$.

(4) Every fixed point of $T$ is an end point of $S$ and every end point of $S$ is a fixed point of $T$. Hence $\operatorname{Fix}(T)=$ $\operatorname{End}(S)$.

So, all the conditions of Theorem 15 are satisfied and hence by application of the Theorem 15 we have required proof.

Theorem 20. Let $(X, d)$ be a complete metric space and $T$ : $X \rightarrow X$ be a generalized almost G-contraction. Suppose that the triple $(X, d, G)$ is regular. Then the following statements hold:

(1) For any $x \in X^{T}(G),\left.T\right|_{[x]_{\widetilde{G}}}$ has a fixed point.

(2) If $X^{T}(G) \neq \emptyset$ and $G$ is weakly connected, then $T$ has a fixed point in $X$.

(3) If $X^{*}:=\bigcup\left\{[x]_{\widetilde{G}}: x \in X^{T}(G)\right\}$, then $\left.T\right|_{X^{*}}$ has a fixed point.

(4) $\operatorname{Fix}(T) \neq \emptyset$ if and only if $X^{T}(G) \neq \emptyset$.

Theorem 21. Let $(X, d)$ be a complete metric space and $T$ : $X \rightarrow X$ be a generalized almost $G$-contraction. Suppose that the triple $(X, d, G)$ is regular. Then the following statements hold:

(1) For any $x \in X_{T}^{T}(G),\left.T\right|_{[x]_{\widetilde{G}}}$ has a fixed point.

(2) If $X_{T}^{T}(G) \neq \emptyset$ and $G$ is weakly connected, then $T$ has a fixed point in $X$.
(3) If $X^{*}:=\bigcup\left\{[x]_{\widetilde{G}}: x \in X_{T}^{T}(G)\right\}$, then $\left.T\right|_{X^{*}}$ has a fixed point.

(4) $\operatorname{Fix}(T) \neq \emptyset$ if and only if $X_{T}^{T}(G) \neq \emptyset$.

Example 22. Let $X=[0,1] \cup[a, b]$, where $a, b \in \mathbb{R}$ with $\sqrt{2}<$ $a<b$, and let $G$ be a directed graph with $V(G)=X$ and $E(G)=\{(x, y): x, y \in[0,1]$ and $x \geq y\} \cup\{(x, y): x, y \in$ $[a, b]$ and $x \leq y\}$. Let $d$ be metric the usual metric on $X$.

Let $T: X \rightarrow X$ be defined as

$$
T x= \begin{cases}\frac{x}{2}, & \text { if } x \in[0,1], \\ x+\frac{1}{x}-\frac{1}{b}, & \text { for } x \in[a, b] .\end{cases}
$$

Let $\alpha \in(0,1)$ be such that $1-1 / b^{2} \leq \alpha<1$ and $L>1$.

Then all the conditions of Theorem 19 are satisfied and it is seen that the set of fixed points of $T$ is nonempty. Here $\operatorname{Fix}(T)=\{0, b\}$.

Remark 23. The results we obtain here are for functions without any continuity requirement. We assume in our results that $(X, d, G)$ is regular. If we omit this condition in our theorem and, instead, assume the function $T$ to be continuous then also the conclusions of the theorem are valid.

\section{Competing Interests}

The authors declare that there is no conflict of interests regarding the publication of this paper.

\section{Authors' Contributions}

The authors contributed equally to this work.

\section{References}

[1] D. W. Boyd and J. S. Wong, "On nonlinear contractions," Proceedings of the American Mathematical Society, vol. 20, pp. 458464, 1969.

[2] A. C. Ran and M. C. Reurings, "A fixed point theorem in partially ordered sets and some applications to matrix equations," Proceedings of the American Mathematical Society, vol. 132, no. 5, pp. 1435-1443, 2004.

[3] J. J. Nieto and R. López, "Contractive mapping theorems in partially ordered sets and applications to ordinary differential equations," Order, vol. 22, no. 3, pp. 223-239, 2005.

[4] J. J. Nieto and R. Rodríguez-López, "Existence and uniqueness of fixed point in partially ordered sets and applications to ordinary differential equations," Acta Mathematica Sinica, vol. 23, no. 12, pp. 2205-2212, 2007.

[5] M. Turinici, "Abstract comparison principles and multivariable Gronwall-Bellman inequalities," Journal of Mathematical Analysis and Applications, vol. 117, no. 1, pp. 100-127, 1986.

[6] B. S. Choudhury, N. Metiya, and M. Postolache, "A generalized weak contraction principle with applications to coupled coincidence point problems," Fixed Point Theory and Applications, vol. 2013, article 152, 2013. 
[7] J. Harjani and K. Sadarangani, "Fixed point theorems for weakly contractive mappings in partially ordered sets," Nonlinear Analysis: Theory, Methods \& Applications, vol. 71, no. 7-8, pp. 34033410,2009

[8] P. Kaushik, S. Kumar, and P. Kumam, "Coupled coincidence point theorems for $\alpha-\psi$-contractive type mappings in partially ordered metric spaces," Fixed Point Theory and Applications, vol. 2013, article 325, 2013.

[9] H. K. Nashine, B. Samet, and C. Vetro, "Monotone generalized nonlinear contractions and fixed point theorems in ordered metric spaces," Mathematical and Computer Modelling, vol. 54, no. 1-2, pp. 712-720, 2011.

[10] I. Beg and A. R. Butt, "Common fixed point for generalized set valued contractions satisfying an implicit relation in partially ordered metric spaces," Mathematical Communications, vol. 15, no. 1, pp. 65-76, 2010.

[11] B. S. Choudhury and N. Metiya, "Multivalued and singlevalued fixed point results in partially ordered metric spaces," Arab Journal of Mathematical Sciences, vol. 17, no. 2, pp. 135-151, 2011.

[12] B. S. Choudhury and N. Metiya, "Fixed point theorems for almost contractions in partially ordered metric spaces," Annali Dell'Universita' Di Ferrara, vol. 58, no. 1, pp. 21-36, 2012.

[13] B. S. Choudhury and N. Metiya, "Coincidence point theorems for a family of multivalued mappings in partially ordered metric spaces," Acta Universitatis Matthiae Belii, Series Mathematics, vol. 21, pp. 13-26, 2013.

[14] S. Hong, "Fixed points of multivalued operators in ordered metric spaces with applications," Nonlinear Analysis: Theory, Methods \& Applications, vol. 72, no. 11, pp. 3929-3942, 2010.

[15] J. Jachymski, "The contraction principle for mappings on a metric space with a graph," Proceedings of the American Mathematical Society, vol. 136, no. 4, pp. 1359-1373, 2008.

[16] M. Abbas, T. Nazir, T. A. Lampert, and S. Radenović, "Common fixed points of set-valued F-contraction mappings on domain of sets endowed with directed graph," Computational and Applied Mathematics, 2016

[17] I. Beg, A. R. Butt, and S. Radojević, "The contraction principle for set valued mappings on a metric space with a graph," Computers \& Mathematics with Applications, vol. 60, no. 5, pp. 12141219, 2010.

[18] F. Bojor, "Fixed point of $\varphi$-contraction in metric spaces endowed with a graph," Annals of the University of Craiova, Mathematics and Computer Science Series, vol. 37, no. 4, pp. 8592, 2010.

[19] F. Bojor, "Fixed point theorems for Reich type contractions on metric spaces with a graph," Nonlinear Analysis: Theory, Methods \& Applications, vol. 75, no. 9, pp. 3895-3901, 2012.

[20] J. Tiammee and S. Suantai, "Coincidence point theorems for graph-preserving multi-valued mappings," Fixed Point Theory and Applications, vol. 2014, article 70, 11 pages, 2014.

[21] B. Fisher, "Common fixed points of mappings and setvalued mappings," Rostock Mathematical Colloquium, vol. 18, pp. 6977, 1981.

[22] S. B. Nadler Jr., "Multi-valued contraction mappings," Pacific Journal of Mathematics, vol. 30, pp. 475-488, 1969.

[23] L. B. Ćirić and J. S. Ume, "Some common fixed point theorems for weakly compatible mappings," Journal of Mathematical Analysis and Applications, vol. 314, no. 2, pp. 488-499, 2006.

[24] B. Fisher and S. Sessa, "Two common fixed point theorems for weakly commuting mappings," Periodica Mathematica Hungarica, vol. 20, no. 3, pp. 207-218, 1989.
[25] V. Berinde, "Approximating fixed points of weak contractions using the Picard iteration," Nonlinear Analysis Forum, vol. 9, no. 1, pp. 43-53, 2004.

[26] R. Kannan, "Some results on fixed points," Bulletin of Calcutta Mathematical Society, vol. 10, pp. 71-76, 1968.

[27] T. Zamfirescu, "Fix point theorems in metric spaces," Archiv der Mathematik, vol. 23, pp. 292-298, 1972.

[28] G. V. Babu, M. L. Sandhya, and M. V. Kameswari, "A note on a fixed point theorem of Berinde on weak contractions," Carpathian Journal of Mathematics, vol. 24, no. 1, pp. 8-12, 2008.

[29] V. Berinde and M. Păcurar, "Fixed points and continuity of almost contractions," Fixed Point Theory, vol. 9, no. 1, pp. 2334, 2008.

[30] L. Cirić, M. Abbas, R. Saadati, and N. Hussain, "Common fixed points of almost generalized contractive mappings in ordered metric spaces," Applied Mathematics and Computation, vol. 217, no. 12, pp. 5784-5789, 2011.

[31] M. Păcurar, "Sequences of almost contractions and fixed points," Carpathian Journal of Mathematics, vol. 24, no. 2, pp. 101-109, 2008. 


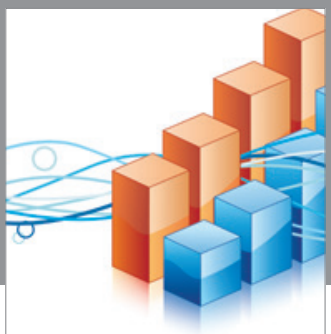

Advances in

Operations Research

vatem alat4

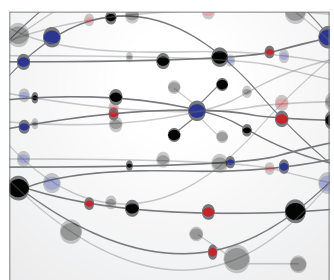

\section{The Scientific} World Journal
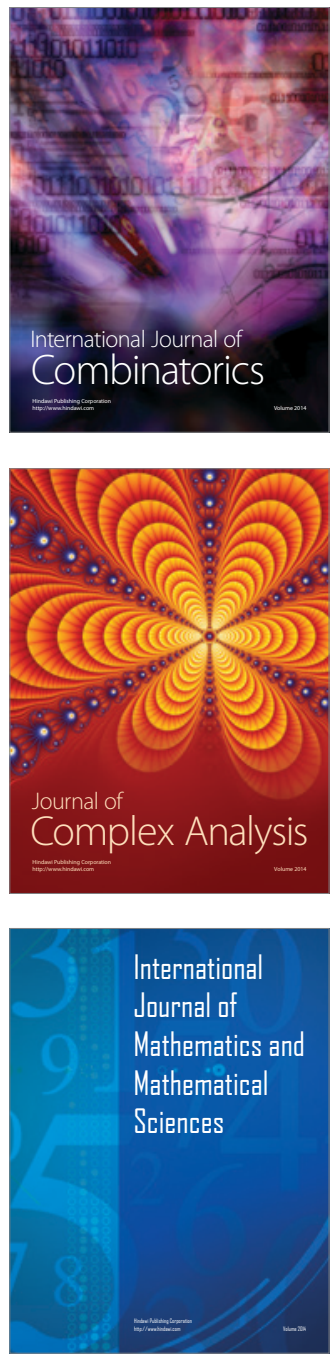
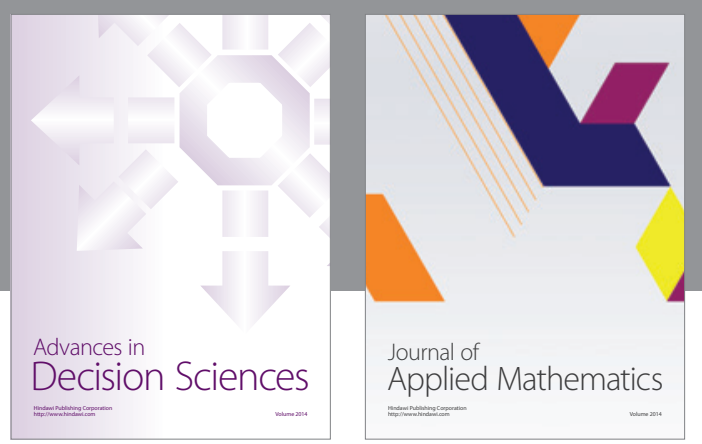

Algebra

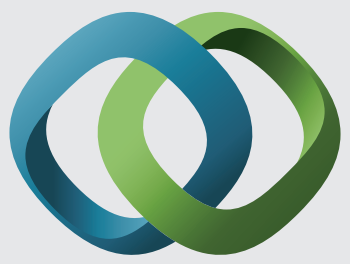

\section{Hindawi}

Submit your manuscripts at

http://www.hindawi.com
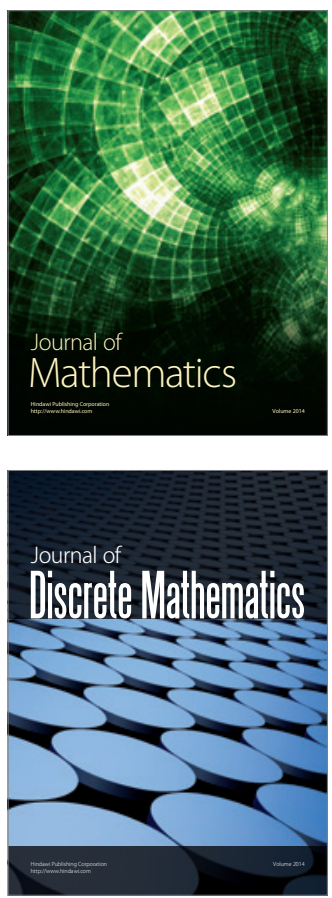

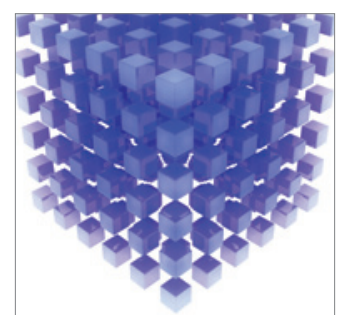

Mathematical Problems in Engineering
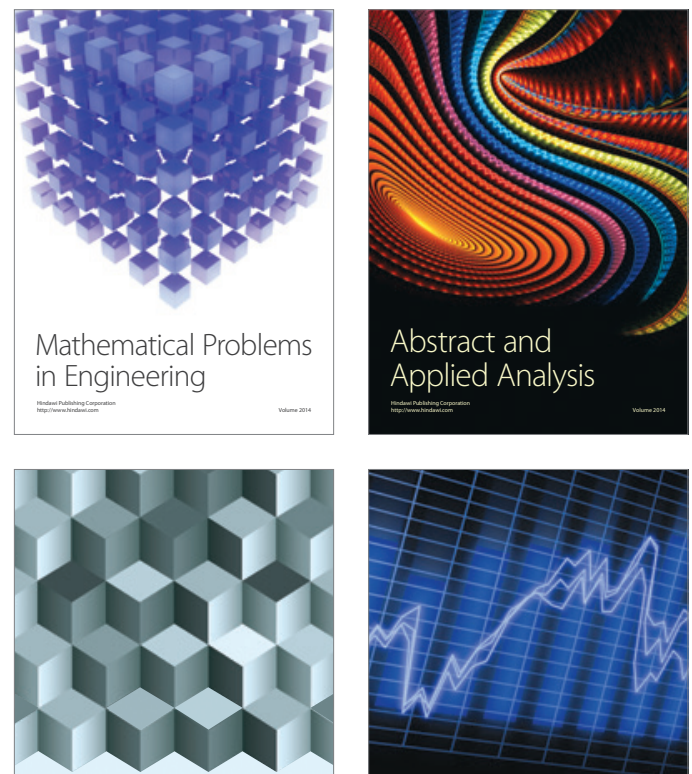

Journal of

Function Spaces

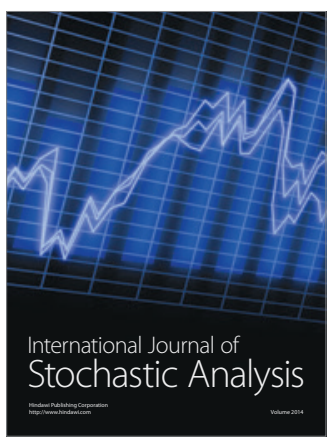

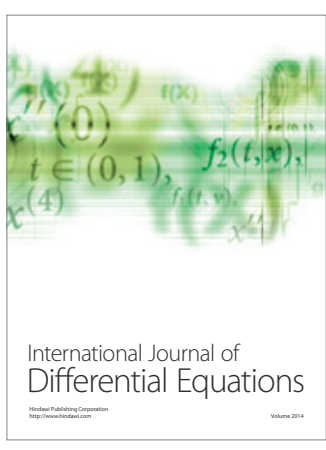
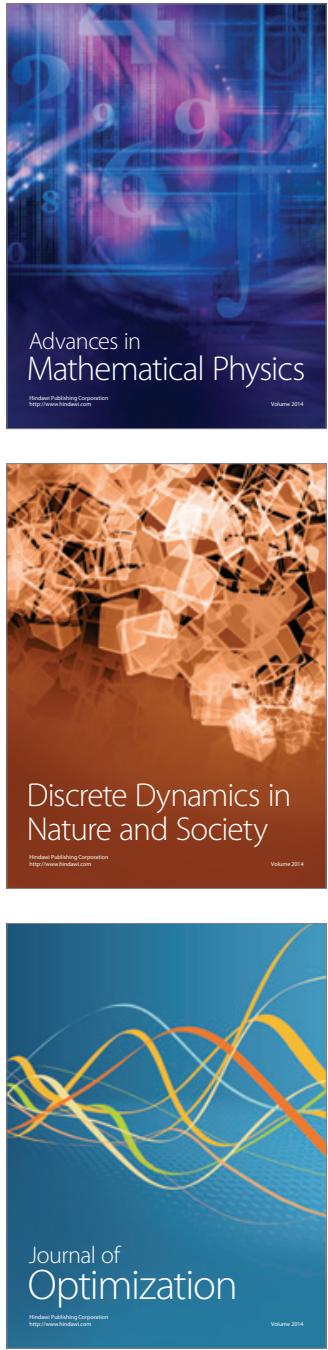